an ALC $<1.0 \times 10^{9} / \mathrm{l}$ tended to do better does not discredit the role of the host immunity in affecting survival in patients treated with RIT, as it might be the possibility that in their low ALC group there was higher NK cells numbers explaining the observed better outcome. Therefore, we agree with the authors that prospective studies are warranted to address these issues.

\section{LF Porrata ${ }^{1}$, K Ristow $^{1}$, TE Witzig ${ }^{1}, \mathrm{~N}$ Tuinistra $^{2}$ TM Habermann ${ }^{1}$, DJ Inwards ${ }^{1}$, SM Ansell ${ }^{1}$, IN Micallef ${ }^{1}$, PB Johnston ${ }^{1}$ and $\mathrm{SN}$ Markovic ${ }^{1}$ \\ ${ }^{1}$ Division of Hematology/Department of Medicine, Mayo Clinic College of Medicine, Rochester, MN, USA and \\ ${ }^{2}$ Department of Nuclear Medicine, Mayo Clinic College of Medicine, Rochester, MN, USA E-mail:porrata.Iuis@mayo.edu}

\section{References}

1 Bishton MJ, Hicks RJ, Prince HM, Ritchie DS, Wolf M, Seymour JF. Claimed association of absolute lymphocyte count with therapeutic efficacy of radio-immunotherapy in patients with indolent lymphoma cannot be verified in an independence data set. Leukemia 2008; e-pub ahead of print 15 May 2008; doi:10.1038/leu.2008.116.

2 Porrata LF, Ristow K, Witzig TE, Tunistra N, Habermann TM Inwards DJ et al. Absolute lymphocyte count predicts therapeutic efficacy and survival at the time of radioimmunotherapy in patients with relapsed follicular lymphomas. Leukemia 2007; 21: 2554-2556.

3 Porrata LF, Markovic SN. Timely reconstitution of immune competence affects clinical outcome following autologous stem cell transplantation. Clin Exp Med 2004; 4: 78-85.

4 Siddiqui M, Ristoe K, Markovic SN, Witzig TE, Habermann TM, Colgan JP et al. Absolute lymphocyte count predicts overall survival in follicular lymphomas. $\mathrm{Br} /$ Haematol 2006; 134: 596-601.

5 Behl D, Porrata LF, Markovic SN, Letendre L, Pruthi RK, Hook CC et al. Absolute lymphocyte count recovery after induction chemotherapy predicts superior survival in acute myelogenous leukemia. Leukemia 2006; 20: 29-34.

6 De Angulo G, Hernandez M, Morales-Arias J, Herzog CE, Anderson $\mathrm{P}$, Wolff $\mathrm{J}$ et al. Early lymphocyte recovery as a prognostic indicator for high-risk Ewing sarcoma. J Pediatr Hematol/Oncol 2007; 29: 48-52.

7 De Angulo G, Yuen C, Palla SL, Anderson PM, Zweidler-Mckay PA. Absolute lymphocyte count is a novel prognostic indicator in ALL and AML: implication for risk stratification and future studies. Cancer 2008; 112: 407-415.

$8 \mathrm{Kim} \mathrm{DH}$, Baek JH, Chae YS, Kim YK, Kim HJ, Park YH et al. Absolute lymphocyte counts predicts response to chemotherapy and survival in diffuse large B-cell lymphoma. Leukemia 2007; 21: $2227-2230$

9 Plonquet A, Haioun C, Jais JP, Debard AL, Salles G, Bene MC et al. Peripheral blood natural killer cell count is associated with clinical outcome in patients with aalPI 2-3 diffuse large B-cell lymphoma. Ann Oncol 2007; 18: 1209-1215.

\title{
Oncolytic virotherapy for multiple myeloma using a tumour-specific double-deleted vaccinia virus
}

Leukemia (2008) 22, 2261-2264; doi:10.1038/leu.2008.120;

published online 29 May 2008

Oncolytic virotherapy is a tumour-specific strategy, in which viruses selectively kill cancer cells, either through targeted alterations in the cancer cell, ${ }^{1,2}$ viral deletions, ${ }^{3,4}$ tissue-specific transcriptional control ${ }^{5}$ or tumour-specific receptors. ${ }^{6,7}$ Normal cells are generally left intact. Haematologic malignancies such as multiple myeloma (MM) are particularly attractive for this approach as patients generally have less bulky disease and may have immune system dysfunction favouring viral replication. To date, $\mathrm{MM}$ has demonstrated pre-clinical responses to oncolytic measles virus, ${ }^{6,7}$ vesicular stomatitis virus ${ }^{8}$ and coxsackievirus A21. ${ }^{9}$ Our lab has previously developed an attenuated, recombinant double-deleted vaccinia virus (VV) that infects, replicates and expresses genes preferentially in rapidly dividing tumour cells. ${ }^{4}$ We asked whether $\mathrm{VV}$ would be an effective oncolytic virus against MM.

Initially, fluorescence-activated cell sorting analysis was used to quantitate the percentage of enhanced green fluorescent protein (EGFP)-positive MM cells after infection with an EGFPexpressing VV. All five cells lines were infected (Figure 1a). After $72 \mathrm{~h}, \mathrm{RPMI} 8226$ was most efficiently infected and U266 was the least efficient. To confirm that $\mathrm{VV}$ infection and gene expression in $M M$ cell lines led to oncolysis and cell death, $M M$ cell lines were infected with VV at a multiplicity of infection (MOI) of 1, and MTT assays were performed (Figure 1b). Compared to mockinfected controls (100\% viable), all cell lines showed decreased viability from 17 to $29 \%$ at $72 \mathrm{~h}$. To validate the MTT assays, a trypan blue exclusion assay was performed with similar results (Supplementary Figure S1). In vitro oncolysis, as measured by an assay to detect cytopathic effects, was visible by $48 \mathrm{~h}$ (Figure 1c) after infection for the majority of cell lines. This was delayed for the U266 and H929 cell lines suggesting that these cell lines are less susceptible to VV-mediated cell killing though both were infected and express EGFP (Figure 1c, bottom panel).

As many anti-myeloma therapeutics act through the induction of apoptosis in MM cells, we asked whether VV-mediated cell death was due to a similar mechanism. Annexin $\mathrm{V}$ staining was used to quantitate the number of cells undergoing apoptosis. Compared to mock infection alone, VV infection induced apoptosis in four of five cell lines by $72 \mathrm{~h}$ (Supplementary Figure S2). The number of necrotic cells was also increased over mock infection.

We next evaluated VV-mediated oncolysis in established tumours after systemic delivery. My5 subcutaneous xenograftbearing mice were treated with systemic (intraperitoneal, $10^{9}$ plaque-forming unit (PFU) per mouse) $\mathrm{VV}$ or vehicle control. After treatment with a single dose of $\mathrm{VV}$, the growth of My5 (Figure 2a) xenografts was significantly $(P<0.0001)$ inhibited by the treatment compared to vehicle control. In addition, My5-bearing mice re-treated with a second dose of $\mathrm{V} V$ showed further tumour regression. Selected mice underwent fluorescent imaging at various time points after infection with the EGFPexpressing VV. Viral-mediated EGFP expression was minimal 6 days after $\mathrm{VV}$ infection, but slowly spread throughout the tumour and peaked 3 weeks post-infection (Figure $2 b$ ). 


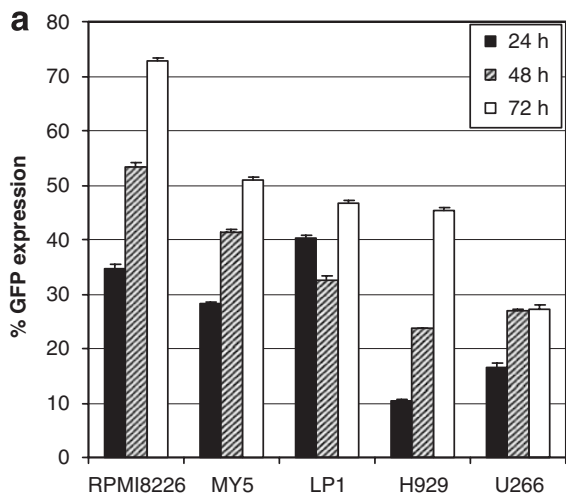

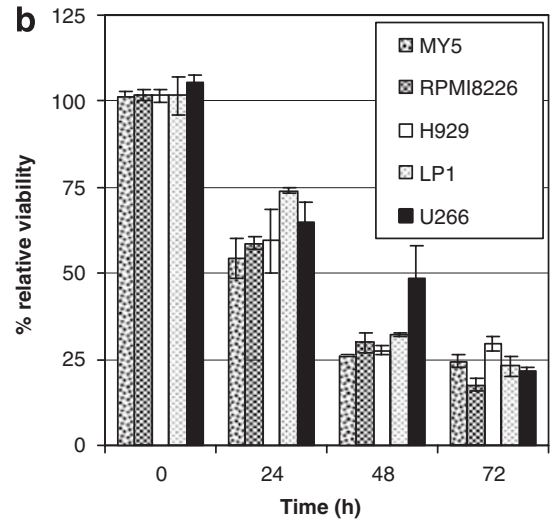

c
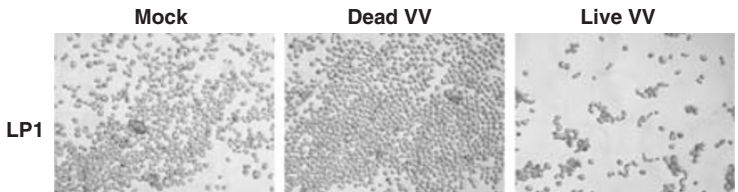

RPMI8226
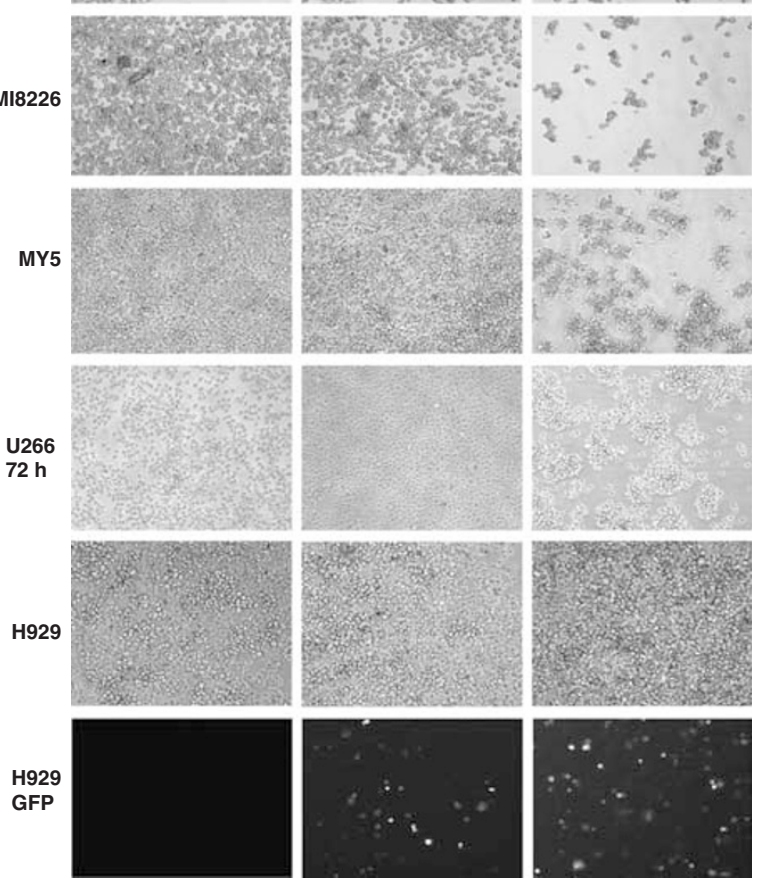

Figure 1 Vaccinia virus infects and kills myeloma cell lines. (a) Fluorescence-activated cell sorting results for EGFP expression in MM cell lines, 24 (black bar), 48 (striped bar) and $72 \mathrm{~h}$ (white bar) after infection with an EGFP-expressing VV at an MOI of 1 . All samples were performed in triplicate. Error bar =s.d. (b) MTT assay of MM cell lines was performed after infection with VV at an MOI of 1 . Mean percentage viability over time ( \pm s.d.) is shown relative to mock-infected controls. Each experiment was performed in triplicate. (c) Cytopathic effects on $\mathrm{MM}$ cell lines $48 \mathrm{~h}(72 \mathrm{~h}$ for U266) after treatment with vehicle alone (left), ultraviolet-killed VV (middle) or live VV (right) at an MOI of 1. GFP filter is also shown for H929. $\mathrm{MOI}$, multiplicity of infection; MM, multiple myeloma; VV, vaccinia virus. EGFP, enhanced green fluorescent protein.

In the setting of haematologic malignancies, where malignant cells are wide spread, the ability to deliver a systemic therapy that can target all tumour cells is critical. The most remarkable response we saw was in the treatment of a model of systemic MM. ${ }^{10}$ This is a murine model that closely resembles the human disease, in which MM cells lodge in the spine and skull after intravenous delivery. In untreated mice, this model results in paralysis within 3 weeks. Nonobese diabetic-severe combined immunodeficient (NOD-SCID) mice were irradiated and injected with RPMI8226 cells via tail vein. Groups of mice were treated with intraperitoneal VV or vehicle control on day 3 or 7 after tumour injection. VV was very effective in this setting. As seen in Figure 2c, mice receiving the vehicle control died with a median survival of 15 days. Mice receiving $\mathrm{V}$ on day 7 had an intermediate survival of 26 days (median). Of mice treated 3 days after tumour injection, $80 \%$ were alive and well more than 100 days after tumour injection $(P<0.01)$.

To confirm that systemic delivery of $\mathrm{V}$ resulted in tumourspecific viral replication, other mouse tissues were analysed. Twenty-one days after VV injection, mice were euthanized and tissues analysed for live virus content by plaque assay. In agreement with the EGFP imaging, tumour was the site of maximum viral replication with a 4-log increase over the next highest tissue (ovary) and a 3-log increase over the total injected dose, confirming viral replication in the tumour (Supplementary Figure S3). Minimal live virus was recovered from other organs. Tissues from these mice were also examined by a pathologist (CM) for evidence of any histopathological change in selected nontumour tissues. With the exception of ovary (Figure 2d), which had evidence of necrosis from presumed viral replication, no other tissues showed any evidence of histopathological lesions or changes consistent with toxicity or adverse reaction. In particular, brain, liver and bowel were normal (Figure 2d). No evidence of histopathological changes in the bone marrow were apparent.

Finally, we investigated the ability of $\mathrm{VV}$ to infect primary bone marrow mononuclear cells of patients with MM and peripheral blood mononuclear cells (PBMCs) from normal volunteers. Cells were infected with a low viral $\mathrm{MOI}$, and significant rates of infection were seen in the malignant MM cells. As seen in Figure 3a (and Supplementary Table S1), 50 $75 \%$ of the CD138 + cells expressed VV-mediated EGFP $72 \mathrm{~h}$ after infection, in the majority of MM patients tested. Only one patient had a low level (4\%) of CD138+ cell infection. Surrounding normal cells were relatively uninfected. PBMCs of normal donors demonstrated very low levels of infectivity. Seventy-two hours postinfection, minimal numbers of cells showed viral EGFP expression (Figure 3b). Fluorescenceactivated cell sorting analysis to determine the cell populations that were infected showed an overall infectivity of normal PBMCs of $1-2 \%$ after $72 \mathrm{~h}$; however, specific populations of lymphocytes were much less (CD3 + : $0.03 \pm 0.03 \%$; CD19+: $0.16 \pm 0.12 \%$; Figure 3c).

Here we have presented the first evidence that MM responds well to systemic VV therapy and should be considered for 


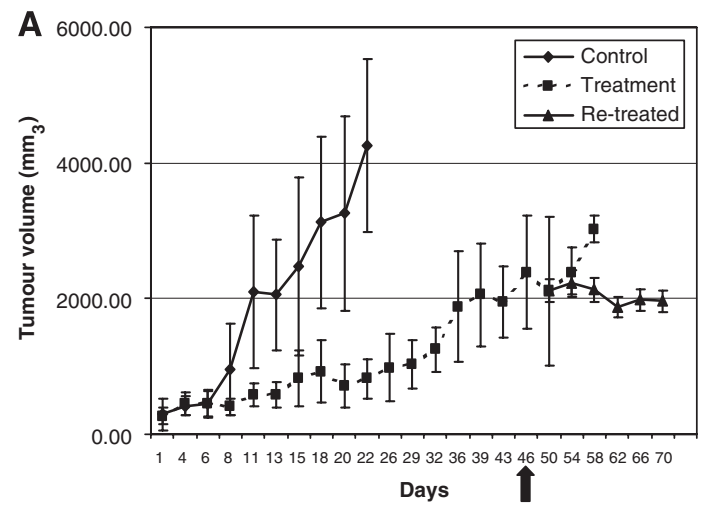

B
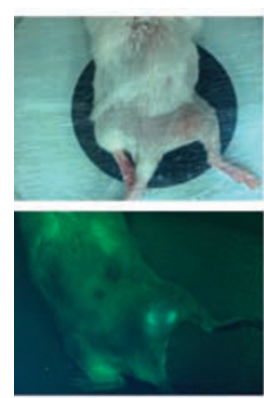

Day 18
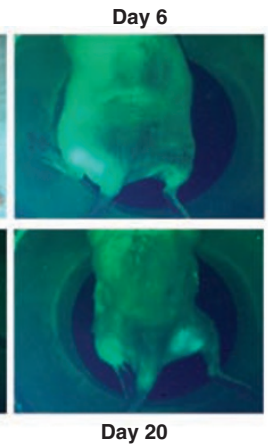

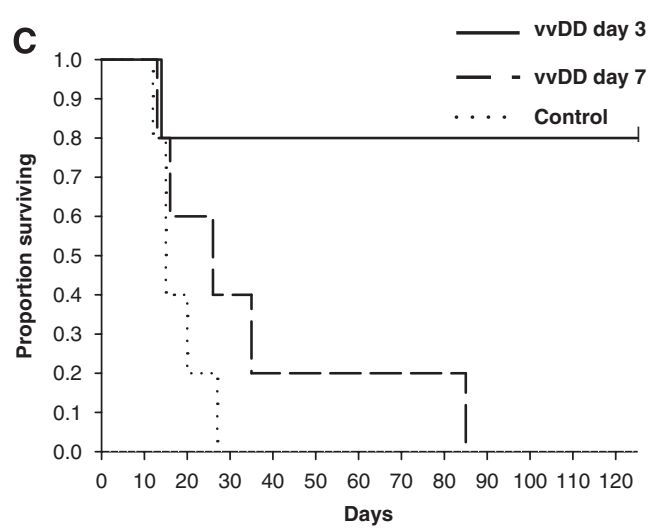

Figure 2 Double-deleted vaccinia virus inhibits growth of MM xenografts. (A) Female NOD-SCID mice were injected with My5 cells SQ in the right flank. When tumours developed, mice were administered either $10^{9} \mathrm{PFU}$ of VV or vehicle control $(n=8)$ via i.p. injection. Tumours were measured two times per week and volumes were calculated as $(\mathrm{L} \times \mathrm{W} \times \mathrm{W}) / 2$. Half $(n=4)$ of the previously treated mice received a second viral injection $\left(10^{9} \mathrm{PFU}\right)$ on day 46 (arrow). Error bars =s.d. (B) A mouse from A was serially imaged to follow viral-mediated EGFP expression over time. Images were obtained using a white light filter (top left image) or fluorescent filter (magnification, $\times 0.8$ ). Fluorescent images taken 6,18 and 21 days after viral injection are shown. (C) Female NOD-SCID mice were irradiated with whole-body $\gamma$-irradiation ( $300 \mathrm{rad}) 24 \mathrm{~h}$ prior to injection with RPMI8226 cells via tail vein. After 3 (solid line) or 7 (dashed line) days, mice were injected with $10^{9}$ PFU of VV or vehicle control (dotted line) i.p. ( $n=5$ per group) and followed for hind limb paralysis. (D) Mice bearing My5 xenografts received $10^{9}$ PFU of i.p. VV. After 21 days, mice were euthanized $(n=3)$ and tumours and normal organs were harvested, formalin-fixed and paraffin-embedded for examination. Histopathological exam revealed normal liver (a), brain (b) and bowel (c). Follicular necrosis was noted in the ovaries (d). Normal bone marrow morphology was seen in both treated animals $(\mathbf{f})$ and controls $(\mathbf{e})$. (magnification, $\times 200$ ). I.p., intraperitoneal; MM, multiple myeloma; PFU, plaque-forming unit; $\mathrm{VV}$, vaccinia virus.

further development. Recent strategies for MM have focused on targeting the malignant cells themselves while avoiding the toxicities associated with traditional chemotherapeutics. We found that VV kills the malignant plasma cells very well, and the tumour specificity of a double-deleted VV is maintained in models of MM. Although this tumour specificity is thought to be due to substrates (such as thymidine triphosphate) present in dividing cancer cells, ${ }^{4} \mathrm{MM}$ cells may provide an added advantage to $\mathrm{V} \mathrm{V}$ replication.

Toxicity to normal tissues is a concern when using a replicating oncolytic virus; however, no toxicities were observed in this study. In fact the 4-log difference in viral replication noted between the tumour and normal tissues provides a wide therapeutic window within which to treat these patients.

One remaining variable is the robust immune response generated to $\mathrm{VV},{ }^{11}$ which may limit effective treatment to one dose. The immune response may also provide a benefit in that tumour cells infected with the virus will be lysed and can potentially present tumour-specific antigens for recognition by the immune system. Given the enhanced in vivo versus in vitro responses seen, we expect that some degree of the tumour response will prove to be immune-mediated.

\section{Acknowledgements}

This work was supported by the University Health Network Gene Therapy Clinical Research Program (JAM), the Leukemia and Lymphoma Society of Canada (JAM) and an NCIC Terry Fox Program Project Grant (AKS).

H Deng ${ }^{1}, \mathrm{~N}$ Tang ${ }^{1}, \mathrm{AE}$ Stief ${ }^{1}, \mathrm{~N}$ Mehta ${ }^{1}, \mathrm{E}$ Baig $^{1}, \mathrm{R} \mathrm{Head}^{1}$, G Sleep ${ }^{1}$, X-Z Yang ${ }^{2}$, C McKerlie ${ }^{3,4}$, S Trudel $^{2,5}$, AK Stewart ${ }^{2}$ and JA' MCCart ${ }^{1,6,7,8}$

${ }^{1}$ Division of Experimental Therapeutics, Toronto General Research Institute, Toronto, Canada; ${ }^{2}$ Department of Medical Oncology and Hematology, University Health Network, Toronto, Canada;

${ }^{3}$ Centre For Modeling Human Disease, Samuel Lunenfeld Research Institute, Mount Sinai Hospital, Toronto, Canada;

${ }^{4}$ Department of Laboratory Medicine and Pathobiology, University of Toronto, Toronto, Canada; ${ }^{5}$ Department of Medicine, University of Toronto, Toronto, Canada; ${ }^{6}$ Department of Surgery, Mount Sinai Hospital, Toronto, Canada;

7 Department of Surgical Oncology, University Health Network, Toronto, Canada and

${ }^{8}$ Department of Surgery, University of Toronto, Toronto, Canada E-mail: amccart@uhnres.utoronto.ca 


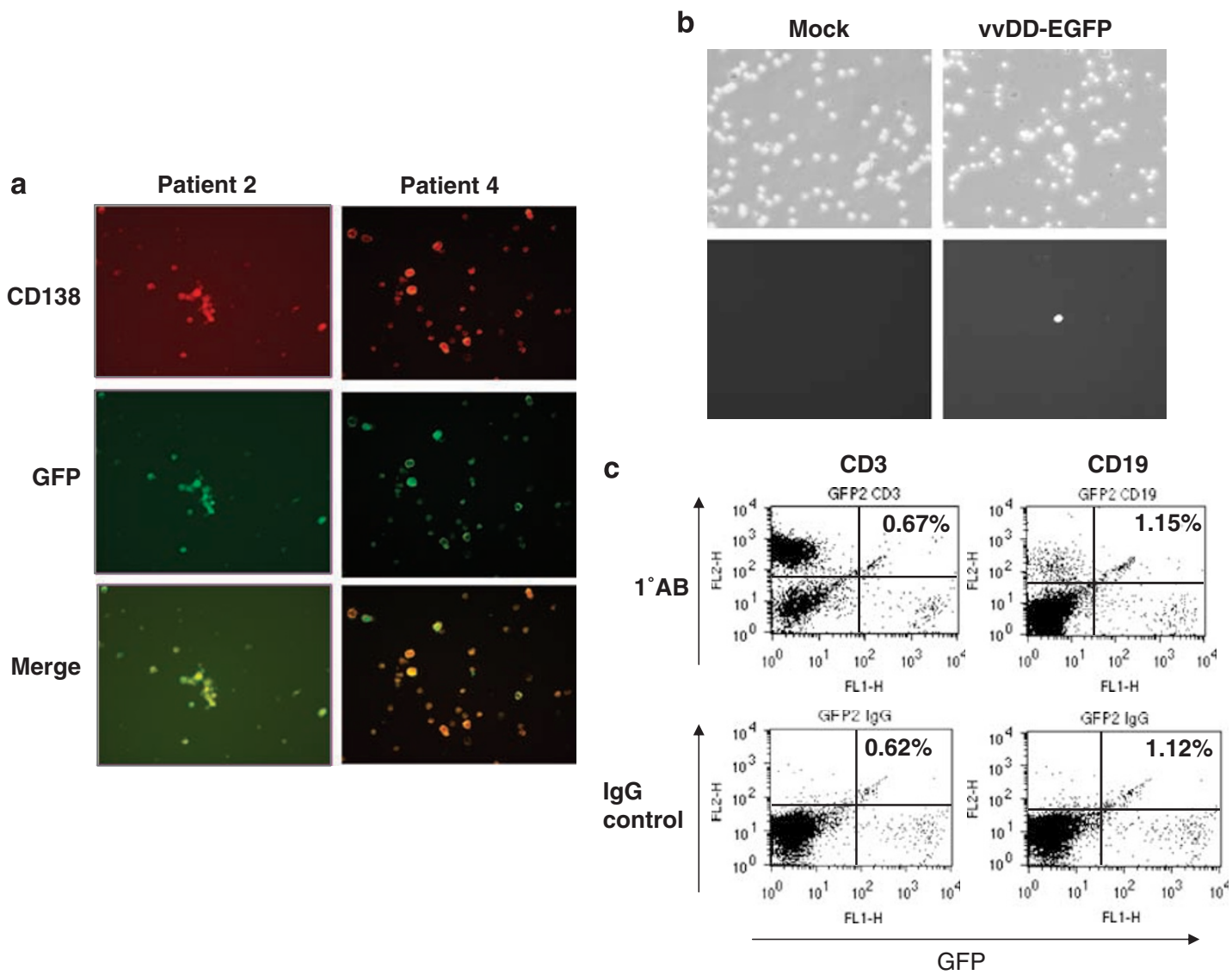

Figure 3 Vaccinia virus infects primary MM cells but not normal PBMCs. (a) Representative images (magnification, $20 \times$ ) of bone marrow mononuclear cells from MM patient nos. 2 and 4 after staining with a PC5-conjugated antibody to CD138. CD138+ cells (top row) and VVinfected EGFP + cells (middle row) are merged (bottom row) to show that the majority of positively stained cells co-localize. (b) PBMCs from a normal volunteer were infected with VV expressing EGFP at an MOI of 1 . Representative photographs (magnification, $20 \times$ ) were taken after $72 \mathrm{~h}$. (c) Representative fluorescence-activated cell sorting plot from normal donor PBMCs infected with VV at an MOI of 1 . After $72 \mathrm{~h}$, cells were stained with anti-CD3 (top left) or anti-CD19 (top right) and analysed by flow cytometry. Respective isotype controls are shown in bottom panels. MOI, multiplicity of infection; MM, multiple myeloma; PBMCs, peripheral blood mononuclear cells; VV, vaccinia virus.

\section{References}

1 Coffey MC, Strong JE, Forsyth PA, Lee PW. Reovirus therapy of tumors with activated Ras pathway. Science 1998; 282: 1332-1334.

2 Stojdl DF, Lichty B, Knowles S, Marius R, Atkins H, Sonenberg N et al. Exploiting tumor-specific defects in the interferon pathway with a previously unknown oncolytic virus. Nature Med 2000; 6: 821-825.

3 Martuza RL, Malick A, Markert JM, Ruffner KL, Coen DM. Experimental therapy of human glioma by means of a genetically engineered virus mutant. Science 1991; 252: 854-856.

4 McCart JA, Ward JM, Lee J, Hu Y, Alexander HR, Libutti SK et al. Systemic cancer therapy with a tumor selective vaccinia virus mutant lacking thymidine kinase and vaccinia growth factor genes. Cancer Res 2001; 61: 8751-8757.

5 Jounaidi Y, Doloff JC, Waxman DJ. Conditionally replicating adenoviruses for cancer treatment. Curr Cancer Drug Targets 2007; 7: 285-301.
6 Peng KW, Ahmann GJ, Pham L, Greipp PR, Russell SJ. Systemic therapy of myeloma xenografts by an attenuated measles virus. Blood 2001; 98: 2002-2007.

7 Peng KW, Donovan KA, Schneider U, Cattaneo R, Lust JA, Russell SJ. Oncolytic measles virus displaying a single chain antibody against CD38, a myeloma cell marker. Blood 2001; 101: 2557-2562.

8 Lichty BD, Stojdl DF, Taylor RA, Miller L, Frenkel I, Atkins $\mathrm{H}$ et al. Vesicular stomatitis virus: a potential therapeutic virus for the treatment of hematological malignancy. Hum Gene Ther 2004; 15: 821-831.

9 Au GG, Lincz LF, Enno A, Shafren DR. Oncolytic Coxsackievirus A21 as a novel therapy for multiple myeloma. Br J Haematol 2007; 137: 133-141.

10 Mitsiades CS, Mitsiades NS, Bronson RT, Chauhan D, Munshi N, Treon SP et al. Fluorescence imaging of multiple myeloma cells in a clinically relevant SCID/NOD in vivo model: biologic and clinical implications. Cancer Res 2003; 63: 6689-6696.

11 Moss B. Genetically engineered poxviruses for recombinant gene expression, vaccination, and safety. Proc Natl Acad Sci USA 1996; 93: $11341-11348$.

Supplementary Information accompanies the paper on the Leukemia website (http://www.nature.com/leu) 